\title{
SPACELIKE HYPERSURFACES IN DE SITTER SPACE WITH CONSTANT HIGHER-ORDER MEAN CURVATURE
}

\author{
KAIREN CAI AND HUIQUN XU
}

Received 26 March 2006; Accepted 26 March 2006

The authors apply the generalized Minkowski formula to set up a spherical theorem. It is shown that a compact connected hypersurface with positive constant higher-order mean curvature $H_{r}$ for some fixed $r, 1 \leq r \leq n$, immersed in the de Sitter space $S_{1}^{n+1}$ must be a sphere.

Copyright (c) 2006 Hindawi Publishing Corporation. All rights reserved.

\section{Introduction}

The classical Liebmann theorem states that a connected compact surface with constant Gauss curvature or constant mean curvature in $\mathbb{R}^{3}$ is a sphere. The natural generalizations of the Gauss curvature and mean curvature are the $r$ th mean curvature $H_{r}, r=1, \ldots, n$, which are defined as the $r$ th elementary symmetric polynomial in the principal curvatures of $M$. Later many authors $[1,4,5,7,8]$ have generalized Liebmann theorem to the cases of hypersurfaces with constant higher-order mean curvature in the Euclidian space, hyperbolic space, the sphere, and so on. A significant result due to Ros [8] is that a compact hypersurface with the $r$ th constant mean curvature $H_{r}$, for some $r=1, \ldots, n$, embedded into the Euclidian space must be a sphere.

The purpose of this note is to prove a spherical theorem of the Liebmann type for the compact spacelike hypersurface immersed in the de Sitter space by setting up a generalized Minkowski formula. The main result is the following.

Theorem 1.1. Let $M$ be a compact connected hypersurface immersed in the de Sitter space $S_{1}^{n+1}$. If for some fixed $r, 1 \leq r \leq n$, the rth mean curvature $H_{r}$ is a positive constant on $M$, then $M$ is isometric to a sphere.

For the cases of the constant mean curvature and constant scalar curvature, that is, $r=1,2$, the theorem was founded by Montiel [4] and Cheng and Ishikawa [1], respectively. 
2 Spacelike hypersurfaces in de Sitter space

\section{Preliminaries}

Let $\mathbb{R}_{1}^{n+2}$ be the real vector space $\mathbb{R}^{n+2}$ endowed with the Lorentzian metric $\langle\cdot, \cdot \cdot\rangle$ given by

$$
\langle x, y\rangle=-x_{0} y_{0}+\sum_{i=1}^{n+2} x_{i} y_{i}
$$

for $x, y \in \mathbb{R}^{n+2}$. The de Sitter space $S_{1}^{n+1}(c)$ can be defined as the following hyperquadratic:

$$
S_{1}^{n+1}(c)=\left\{\left.x \in \mathbb{R}_{1}^{n+2}|| x\right|^{2}=\frac{1}{c}, \frac{1}{c}>0\right\}
$$

In this way, the de Sitter space inherits from $\langle\cdot, \cdot\rangle$ a metric which makes it an indefinite Riemannian manifold of constant sectional curvature $c$. If $x \in S_{1}^{n+1}(c)$, we can put

$$
T_{x} S_{1}^{n+1}(c)=\left\{v \in \mathbb{R}_{1}^{n+2} \mid\langle v, x\rangle=0\right\}
$$

Let $\psi: M \rightarrow S_{1}^{n+1}$ be a connected spacelike hypersurface immersed in the de Sitter space with the sectional curvature 1. Following O'Neill [6], the unit normal vector field $N$ for $\psi$ can be viewed as the Gauss map of $M$ :

$$
N: M \longrightarrow\left\{\left.x \in \mathbb{R}_{1}^{n+2}|| x\right|^{2}=-1\right\}
$$

Let $S_{r}: \mathbb{R}^{n} \rightarrow \mathbb{R}, r=1, \ldots, n$, be the normalized $r$ th elementary symmetric function in the variables $y_{1}, \ldots, y_{n}$. For $r=1, \ldots, n$, we denote by $C_{r}$ the connected component of the set $\left\{y \in \mathbb{R}^{n} \mid S_{r}(y)>0\right\}$ containing the vector $y=(1, \ldots, 1)$. Notice that every vector $\left(y_{1}, \ldots, y_{n}\right)$ with all its components greater than zero lies in each $C_{r}$. We derive the following two lemmas, which will be needed for the proof of the theorem.

Lemma 2.1 [3]. (i) If $r \geq k$, then $C_{r} \subset C_{k}$; (ii) for $y \in C_{r}$,

$$
S_{r}^{1 / r} \leq S_{r-1}^{1 / r-1} \leq \cdots \leq S_{2}^{1 / 2} \leq S_{1}
$$

Lemma 2.2 (Minkowski formula). Let $\psi: M \rightarrow S_{1}^{n+1} \subset \mathbb{R}_{1}^{n+2}$ be a connected spacelike hypersurface immersed in de Sitter space $S_{1}^{n+1}$. For the rth mean curvature $H_{r}$ of $\psi, r=0,1, \ldots$, $n-1$,

$$
\int_{M}\left(H_{r}\langle\psi, a\rangle+H_{r+1}\langle N, a\rangle\right) d V=0
$$

where $H_{0}=1$ and $a \in \mathbb{R}_{1}^{n+1}$ is an arbitrary fixed vector and $N$ is the unit normal vector of $M$.

Proof. The argument is based on the approach of geodesic parallel hypersurfaces in [5]. Let $k_{r}$ and $e_{i}, i=1, \ldots, n$, be the principal curvatures and the principal directions at a point $p \in M$. The $r$ th mean curvature of $\psi$ is defined by the identity

$$
P_{n}(t)=\left(1+t k_{1}\right) \cdots\left(1+t k_{n}\right)=1+\left(\begin{array}{l}
n \\
1
\end{array}\right) H_{1} t+\cdots+\left(\begin{array}{l}
n \\
n
\end{array}\right) H_{n} t^{n}
$$


for all $t \in \mathbb{R}$. Thus $H_{1}=H$ is the mean curvature, $H_{2}=\left(n^{2} H^{2}-S\right) / n(n-1)$, where $S$ is the square length of the second fundamental form and $H_{n}$ is the Gauss-Kronecker curvature of $M$ immersed in $S_{1}^{n+1}$. Let us consider a family of geodesic parallel hypersurfaces $\psi_{t}$ given by

$$
\psi_{t}(p)=\exp _{\psi(p)}(-t N(p))=\cosh t \cdot \psi(p)+\sinh t \cdot N(p) .
$$

Then the unit normal vector field of $\psi_{t}$ with $\left|N_{t}\right|^{2}=-1$ can be written as

$$
N_{t}(p)=-\sinh t \cdot \psi(p)-\cosh t \cdot N(p)
$$

Because we have

$$
\begin{gathered}
\psi_{t_{*}}\left(e_{i}\right)=\left(\cosh t-k_{i} \sinh t\right)\left(e_{i}\right), \\
N_{t_{*}}\left(e_{i}\right)=\left(-\sinh t+k_{i} \cosh t\right)\left(e_{i}\right) ;
\end{gathered}
$$

for the principal directions $\left\{e_{i}\right\}, i=1, \ldots, n$ and $|t|<\varepsilon$, the second fundamental form of $\psi_{t}$ can be expressed as

$$
\begin{aligned}
\sigma_{t}\left(\psi_{t_{*}}\left(e_{i}\right), \psi_{t_{*}}\left(e_{j}\right)\right) & =-\left\langle N_{t_{*}}\left(e_{i}\right), \psi_{t_{*}}\left(e_{j}\right)\right\rangle \\
& =\left(\sinh t-k_{i} \cosh t\right)\left\langle e_{i}, \psi_{t_{*}}\left(e_{j}\right)\right\rangle \\
& =\frac{\sinh t-k_{i} \cosh t}{\cosh t-k_{i} \sinh t}\left\langle\psi_{t_{*}}\left(e_{i}\right), \psi_{t_{*}}\left(e_{j}\right)\right\rangle .
\end{aligned}
$$

Then the mean curvature $H(t)$ of $\psi$ can be expressed as

$$
\begin{aligned}
H(t) & =\frac{1}{n} \sum_{i=1}^{n} k_{i}(t)=\frac{1}{n} \sum_{i=1}^{n} \frac{\tanh t-k_{i}}{1-k_{i} \tanh t} \\
& =\frac{1}{n P_{n}(-\tanh t)} \sum_{i=1}^{n}\left(\tanh t-k_{i}\right) \prod_{j \neq i}\left(1-k_{j} \tanh t\right) .
\end{aligned}
$$

But

$$
\prod_{j \neq i}\left(1-k_{j} \tanh t\right)=n P_{n}(-\tanh t)-\cosh t \sinh t P_{n}^{\prime}(-\tanh t) .
$$

Then we get

$$
H(t)=\tanh t+\frac{P_{n}^{\prime}(-\tanh t)}{n P_{n}(-\tanh t)} .
$$

By the way, we must point out that the formula $\left(7^{\prime}\right)$ in [5] is incorrect because the second term in the right-hand side of the expression of $H(t)$ should be $P_{n}^{\prime}(\tanh t) / n P_{n}(\tanh t)$. The volume element $d V_{t}$ for immersion $\psi_{t}$ can be given by

$$
\begin{aligned}
d V_{t} & =\left(\cosh t-k_{1} \sinh t\right) \cdots\left(\operatorname{conh} t-k_{n} \sinh t\right) d V \\
& =-\operatorname{conh}^{n} t P_{n}(-\tanh t) d V,
\end{aligned}
$$


4 Spacelike hypersurfaces in de Sitter space

where $d V$ is the volume element of $\psi$. It is an easy computation that

$$
\triangle(\langle\psi, a\rangle+H\langle N, a\rangle)=0
$$

where $N$ is a unit normal field of $\psi$ and $a \in \mathbb{R}_{1}^{n+2}$ an arbitrary fixed vector (cf. [4, page 914]). Integrating both sides of (2.16) over the hypersurface $M$ and applying Stoke's theorem, we get

$$
\int_{M}\left(\langle\psi, a\rangle+H_{1}\langle N, a\rangle\right) d V=0
$$

For $\psi_{t},|t|<\varepsilon$, we obtain

$$
\int_{M}\left(\left\langle\psi_{t}, a\right\rangle+H(t)\left\langle N_{t}, a\right\rangle\right) d V_{t}=0
$$

Substituting (2.14) and (2.15) into (2.18), we get

$$
\begin{aligned}
\int_{M}\left\langle\psi_{t}, a\right\rangle+H(t)\left\langle N_{t}, a\right\rangle d V_{t} \\
=\frac{1}{n} \cosh ^{n-1} t \int_{M}\left(\left(n P_{n}(-\tanh t)-\sinh t \cosh t P_{n}^{\prime}(-\tanh t)\right)\langle\psi, a\rangle\right. \\
\left.\quad-\cosh ^{2} t P_{n}^{\prime}(-\tanh t)\langle N, a\rangle\right) d V=0 .
\end{aligned}
$$

By using the expression

$$
\begin{aligned}
& n P_{n}(-\tanh t)-\sinh t \cosh t P_{n}^{\prime}(-\tanh t) \\
& \quad=n+(n-1)\left(\begin{array}{l}
n \\
1
\end{array}\right) H_{1}(-\tanh t)+\cdots+n\left(\begin{array}{c}
n \\
n-1
\end{array}\right) H_{n}(-\tanh t)^{n-1},
\end{aligned}
$$

we obtain

$$
\begin{aligned}
& \int_{M}\left\{\left(n P_{n}(-\tanh t)-\sinh t \cosh t P_{n}^{\prime}(-\tan t)\right)\langle\psi, a\rangle-\operatorname{conh}^{2} t P_{n}^{\prime}(-\tanh t)\langle N, a\rangle\right\} d V \\
& =\sum_{r=1}^{n}(n-r-1)\left(\begin{array}{c}
n \\
r-1
\end{array}\right)(-\tanh t)^{r-1}, \\
& \int_{M}\left(H_{r-1}\left\langle\psi_{t}, a\right\rangle+H_{r}\left\langle N_{t}, a\right\rangle\right) d V=0 .
\end{aligned}
$$

The left-hand side in the equality is a polynomial in the variable tanh $t$. Therefore, all its coefficients are null. This completes the proof of Lemma 2.2.

\section{Proof of Theorem 1.1}

Here we work for the immersed hypersurfaces in $S_{1}^{n+1}$ instead of embedded hypersurfaces because we can only use algebraic inequalities and the integral formula above to complete the proof. Let some $H_{r}$ be a positive constant. Multiplying (2.17) by $H_{r}$ and then 
abstracting from (2.6), we obtain that

$$
\int_{M}\left(H_{1} H_{r}-H_{r+1}\right)\langle N, a\rangle d V=0 .
$$

We know from Newton inequality [2] that $H_{r-1} H_{r+1} \leq H_{r}^{2}$, where the equality implies that $k_{1}=\cdots=k_{n}$. Hence

$$
H_{r-1}\left(H_{1} H_{r}-H_{r+1}\right) \geq H_{r}\left(H_{1} H_{r-1}-H_{r}\right)
$$

It derives from Lemma 2.1 that

$$
0 \leq H_{r}^{1 / r} \leq H_{r-1}^{1 / r-1} \leq \cdots \leq H_{2}^{1 / 2} \leq H_{1} .
$$

Thus we conclude that

$$
H_{r-1}\left(H_{1} H_{r}-H_{r+1}\right) \geq H_{r}\left(H_{1} H_{r_{1}}-H_{r}\right) \geq 0 \text {, }
$$

and if $r \geq 2$, the equalities happen only at umbilical points of $M$. We choose a constant vector $a$ such that $|a|^{2}=-1$ and $a_{0} \leq-1$. Since the normal vector $N$ satisfies $|N|^{2}=-1$, we have $\langle N, a\rangle \geq 1$ on $M$. It follows from (3.1) that

$$
H_{1} H_{r}-H_{r+1}=0 .
$$

Thus, $k_{1}=\cdots=k_{n}, M$ is totally umbilical, and $M$ is isometric to a sphere. This ends the proof of Theorem 1.1.

If there is a convex point on $M$, that is, a point at which $k_{i}>0$, for all $i=1, \ldots, n$, then the constant $r$ th mean curvature $H_{r}$ is positive. By means of the same argument as that of Theorem 1.1, we derive the following.

Corollary 3.1. Let $M$ be a compact connected hypersurface immersed in the de Sitter space $S_{1}^{n+1}$. If for some fixed $r, 1 \leq r \leq n$, the rth mean curvature $H_{r}$ is constant, and there is a convex point on $M$, then $M$ is isometric to a sphere.

\section{Acknowledgment}

The project is supported by the Natural Science Foundation of Zhejiang Provence in China.

\section{References}

[1] Q.-M. Cheng and S. Ishikawa, Spacelike hypersurfaces with constant scalar curvature, Manuscripta Mathematica 95 (1998), no. 4, 499-505.

[2] J. Eells Jr. and J. H. Sampson, Harmonic mappings of Riemannian manifolds, American Journal of Mathematics 86 (1964), 109-160.

[3] L. Gärding, An inequality for hyperbolic polynomials, Journal of Mathematics and Mechanics 8 (1959), 957-965.

[4] S. Montiel, An integral inequality for compact spacelike hypersurfaces in de Sitter space and applications to the case of constant mean curvature, Indiana University Mathematics Journal 37 (1988), no. 4, 909-917. 


\section{Spacelike hypersurfaces in de Sitter space}

[5] S. Montiel and A. Ros, Compact hypersurfaces: the Alexandrov theorem for higher order mean curvatures, Differential Geometry. Proceedings Conference in Honor of Manfredo do Carmo, Pitman Monogr. Surveys Pure Appl. Math., vol. 52, Longman Scientific \& Technical, Harlow, 1991, pp. 279-296.

[6] B. O’Neill, Semi-Riemannian Geometry. With Applications to Relativity, Pure and Applied Mathematics, vol. 103, Academic Press, New York, 1983.

[7] R. C. Reilly, Applications of the Hessian operator in a Riemannian manifold, Indiana University Mathematics Journal 26 (1977), no. 3, 459-472.

[8] A. Ros, Compact hypersurfaces with constant higher order mean curvatures, Revista Matemática Iberoamericana 3 (1987), no. 3-4, 447-453.

Kairen Cai: Department of Mathematics, Hangzhou Teachers College, 222 Wen Yi Road, Hangzhou 310036, China

E-mail addresses: kcai53@hotmail.com ; kairencai408@hzcnc.com

Huiqun Xu: Department of Mathematics, Hangzhou Teachers College, 222 Wen Yi Road, Hangzhou 310036, China

E-mail addresses: huiqunxhu@hotmail.com; xuhuiqun@163.com 


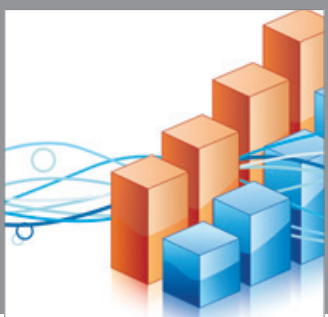

Advances in

Operations Research

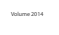

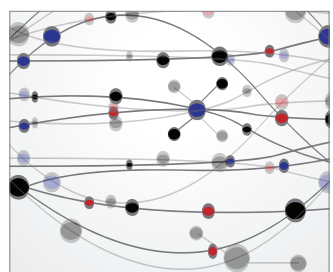

\section{The Scientific} World Journal
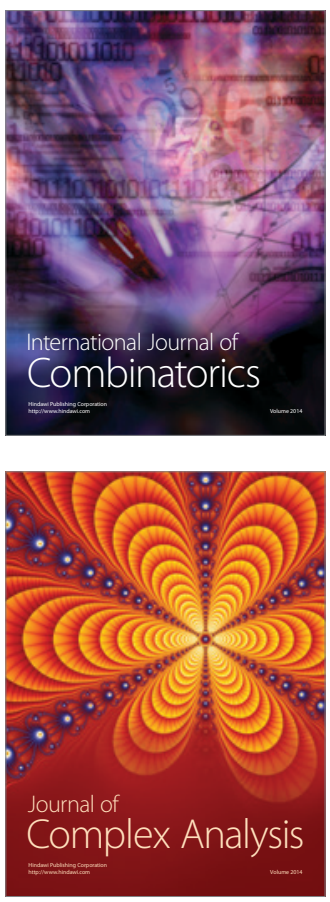

International Journal of

Mathematics and

Mathematical

Sciences
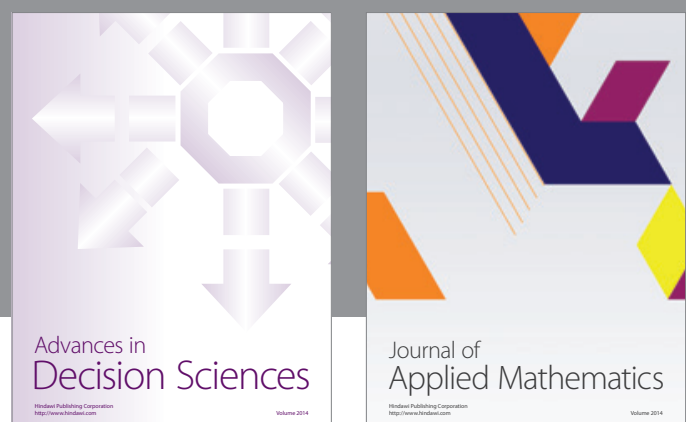

Journal of

Applied Mathematics
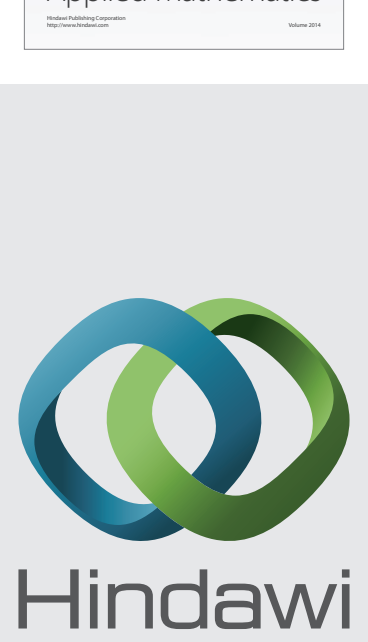

Submit your manuscripts at http://www.hindawi.com
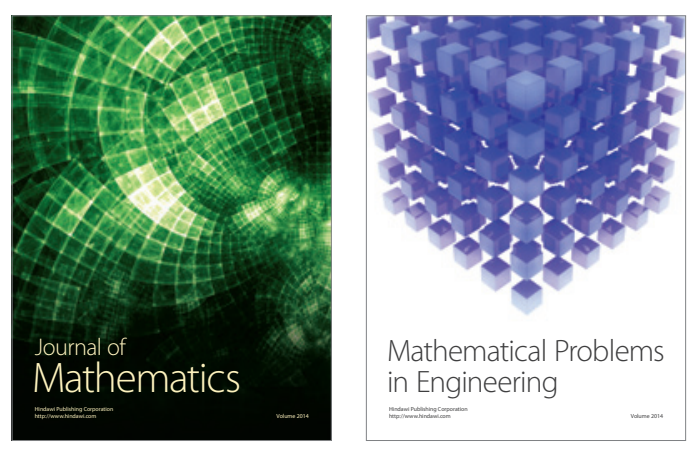

Mathematical Problems in Engineering
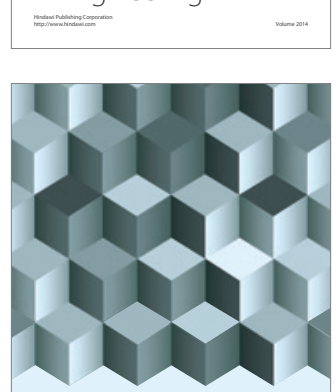

Journal of

Function Spaces
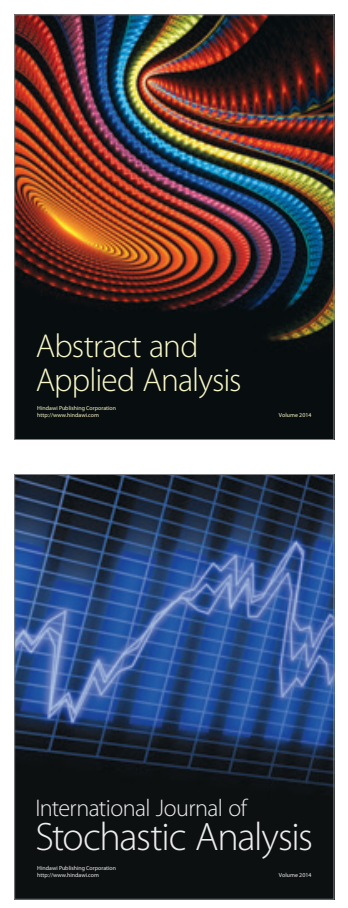

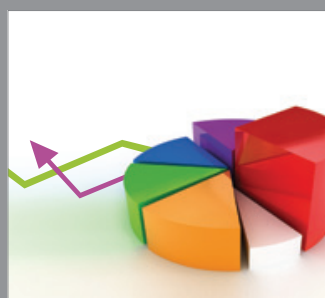

ournal of

Probability and Statistics

Promensencen
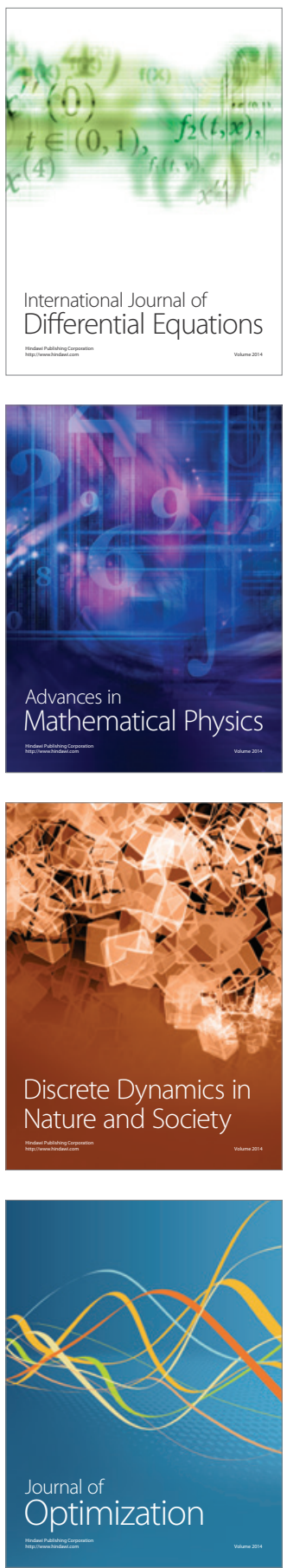